\title{
Semiclassical origin of the spectral gap for transfer operators of partially expanding map.
}

\author{
Frédéric Faure*
}

March 14, 2009

\begin{abstract}
We consider a simple model of partially expanding map on the torus. We study the spectrum of the Ruelle transfer operator and show that in the limit of high frequencies in the neutral direction (this is a semiclassical limit), the spectrum develops a spectral gap, for a generic map. This result has already been obtained by M. Tsujii in [Tsu08a]. The novelty here is that we use semiclassical analysis which provides a different and quite natural description. We show that the transfer operator is a semiclassical operator with a well defined "classical dynamics" on the cotangent space. This classical dynamics has a "trapped set" which is responsible for the Ruelle resonances spectrum. In particular we show that the spectral gap is closely related to a specific dynamical property of this trapped set.
\end{abstract}

\section{Contents}

1 Introduction

2 Model and results 3

2.1 A partially expanding $\operatorname{map} \ldots \ldots \ldots \ldots \ldots \ldots \ldots$

2.2 Transfer operator . . . . . . . . . . . . . . . . .

2.3 The reduced transfer operator . . . . . . . . . . . . . . .

2.4 Main results on the spectrum of the transfer operator $\hat{F}_{\nu} \ldots \ldots \ldots \ldots$

2.5 Spectrum of $\hat{F}$ and dynamical correlation functions . . . . . . . . . . . . .

3 Proof of theorem 1 i on resonances spectrum 9

3.1 Dynamics on the cotangent space $T^{*} S^{1} \ldots \ldots \ldots \ldots \ldots \ldots \ldots$

3.2 The escape function $\ldots \ldots \ldots \ldots \ldots \ldots \ldots$

4 Proof of theorem 2 on spectral gap

4.1 Dynamics on the cotangent space $T^{*} S^{1} \ldots \ldots \ldots \ldots \ldots \ldots$

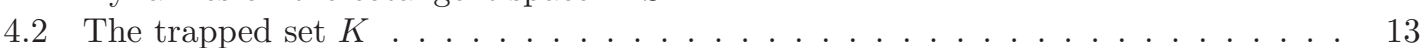

4.3 The escape function $\ldots \ldots \ldots \ldots \ldots \ldots \ldots \ldots \ldots \ldots \ldots \ldots$

A Equivalence classes of dynamics 18

\footnotetext{
*Institut_Fourier.100 rue des Maths, BP74 38402 St Martin d'Hères. frederic.faure@ujf-grenoble.fr http:// ww-fourier.ujf-grenoble.fr/ faure

${ }^{1} 2000$ Mathematics Subject Classification:37D20 hyperbolic systems (expanding, Anosov, Axiom A, etc.) 37C30 Zeta functions, (Ruelle-Frobenius) transfer operators, and other functional analytic techniques in dynamical systems 81Q20 Semi-classical techniques

Keywords: Transfer operator, Ruelle resonances, decay of correlations, Semi-classical analysis.
} 


\section{B Description of the trapped set}

B.1 Dynamics on the cover $\mathbb{R}^{2} \ldots \ldots \ldots \ldots \ldots \ldots \ldots \ldots \ldots$

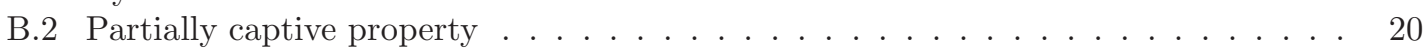

B.3 Fractal aspect of the Trapped set . . . . . . . . . . . . . 22

\section{Introduction}

Chaotic behavior of certain dynamical systems is due to hyperbolicity of the trajectories. This means that the trajectories of two closed initial points will diverge from each other either in the future or in the past (or both). As a result the behavior of an individual trajectory looks like complicated and unpredictable. However evolution of a cloud of points seems more simple: it will spread and equidistributes according to an invariant measure, called an equilibrium measure (or S.R.B. measure). Following this idea, D. Ruelle in the 70' Rue78, Rue86, has shown that instead of considering individual trajectories, it is much more natural to consider evolution of densities under a linear operator called the Ruelle Transfer operator or the Perron Frobenius operator.

For dynamical systems with strong chaotic properties, such as uniformly expanding maps or uniformly hyperbolic maps, Ruelle, Bowen, Fried, Rugh and others, using symbolic dynamics techniques, have shown that the spectrum of the transfer operator has a discrete spectrum of eigenvalues. This spectral description has an important meaning for the dynamics since each eigenvector corresponds to an invariant distribution (up to a time factor). From this spectral characterization of the transfer operator, one can derive other specific properties of the dynamics such as decay of time correlation functions, central limit theorem, mixing, ... In particular a spectral gap implies exponential decay of correlations.

This spectral approach has recently (2002-2005) been improved by M. Blank, S. Gouëzel, G. Keller, C. Liverani BKL02 GL05, Liv05 and V. Baladi and M. Tsujii Bal05, BT07 (see BT07 for some historical remarks), through the construction of functional spaces adapted to the dynamics, independent of any symbolic dynamics. The case of dynamical systems with continuous time is more delicate (see [FMT07] for historical remarks). This is due to the direction of time flow which is neutral (i.e. two nearby points on the same trajectory will not diverge from each other). In 1998 Dolgopyat Dol98, Dol02] showed the exponential decay of correlation functions for certain Anosov flows, using techniques of oscillatory integrals and symbolic dynamics. In 2004 Liverani Liv04 adapted Dolgopyat's ideas to his functional analytic approach, to treat the case of contact Anosov flows. In 2005 M. Tsujii [Tsu08a] obtained an explicit estimate for the spectral gap for the suspension of an expanding map. Then in $2008 \mathrm{M}$. Tsujii [Tsu08b obtained an explicit estimate for the spectral gap, in the case of contact Anosov flows.

Semiclassical approach for transfer operators: It also appeared recently [FR06, FRS08] that for hyperbolic dynamics, the study of transfer operator is naturally a semiclassical problem in the sense that a transfer operator can be considered as a "Fourier integral operator" and using standard tools of semiclassical analysis, some of its spectral properties can be obtained from the study of "the associated classical symplectic dynamics", namely the initial hyperbolic dynamics lifted on the cotangent space (the phase space).

The simple idea behind this, crudely speaking, is that a transfer operator transports a "wave packet" (i.e. localized both in space and in Fourier space) into another wave packet, and this is exactly the characterization of a Fourier integral operator. A wave packet is characterized by a point in phase space (its position and its momentum), hence one is naturally led to study the dynamics in phase space. Moreover, since any function or distribution can be decomposed as a linear superposition of wave packets, the dynamics of wave packets characterizes completely the transfer operators.

Following this approach, in the papers [FR06, FRS08] we studied hyperbolic diffeomorphisms. The aim of the present paper is to show that semiclassical analysis is also well adapted (even better) for hyperbolic systems with neutral direction. We consider here the simplest model: a partially expanding map $f:(x, s) \rightarrow f(x, s)$, i.e. a map on a torus $(x, s) \in S^{1} \times S^{1}$ with an 
expanding direction $\left(x \in S_{x}^{1}\right)$ and a neutral direction $\left(s \in S_{s}^{1}\right)$ (the inverse map $f^{-1}$ is $k$-valued, with $k \geq 2$ ). The results are presented in section 2 . We summarize them in few lines. First in order to reduce the problem and drop out the neutral direction, we use a Fourier analysis in $s \in S_{s}^{1}$ and decompose the transfer operator $\hat{F}$ on $S_{x}^{1} \times S_{s}^{1}$ (defined by $\hat{F} \varphi:=\varphi \circ f$ ) as a collection of transfer operators $\hat{F}_{\nu}$ on the expanding space $S_{x}^{1}$ only, with $\nu \in \mathbb{Z}$ being the Fourier parameter and playing the role of the semiclassical parameter. The semiclassical limit is $|\nu| \rightarrow \infty$.

Then we introduce a (multivalued) map $F_{\nu}$ on the cotangent space $(x, \xi) \in T^{*} S_{x}^{1}$ which is the canonical map associated to the transfer operator $\hat{F}_{\nu}$. The fact that the initial map $f$ is expanding along the space $S_{x}^{1}$ implies that on the cylinder $T^{*} S_{x}^{1}$ trajectories starting from a large enough value of $|\xi|$ escape towards infinity $(|\xi| \rightarrow \infty)$. We define the trapped set as the compact set $K=\lim _{n \rightarrow \infty} F_{\nu}^{-n}\left(K_{0}\right)$ where $K_{0} \subset T^{*} S^{1}$ is an initial large compact set. $K$ contains trajectories which do not escape towards infinity.

Using a standard semiclassical approach (with escape functions on phase space [HS86]) we first show that the operator $\hat{F}_{\nu}$ as a discrete spectrum called Ruelle resonances (we have to consider $\hat{F}_{\nu}$ in Sobolev space of distributions). This is Theorem 1. This result is well known, but the semiclassical approach we use here is new.

Then we show that a specific hypothesis on the trapped set implies that the operator $\hat{F}_{\nu}$ develops a "spectral gap" in the semi-classical limit $\nu \rightarrow \infty$ (i.e. its spectral radius reduces). This is Theorem 2 illustrated on Figure 2. This Theorem is very similar to Theorem 1.1 in Tsu08a. With the semiclassical approach, this result is very intuitive: the basic idea (followed in the proof) is that an initial wave packet $\varphi_{0}$ represented as a point on the trapped set $K$ evolves in several wave packets $\left(\varphi_{j}\right)_{j=1 \rightarrow k}$ under the transfer operator $\hat{F}_{\nu}$, but in general only one wave packet remains on the trapped set $K$ and the $(k-1)$ other ones escape towards infinity. As a result the probability on the trapped set $K$ decays by a factor $1 / k$. This is the origin of the spectral gap at $1 / \sqrt{k}$ on Figure 2.

This work has been supported by "Agence Nationale de la Recherche" under the grant JC05_52556.

\section{Model and results}

\section{$2.1 \quad$ A partially expanding map}

Let $g: S^{1} \rightarrow S^{1}$ be a $C^{\infty}$ diffeomorphism (on $S^{1}:=\mathbb{R} / \mathbb{Z}$ ). $g$ can be written as $g: \mathbb{R} \rightarrow \mathbb{R}$ with $g(x+1)=g(x)+1, \forall x \in \mathbb{R}$. Let $k \in \mathbb{N}, k \geq 2$, and let the map $E: S^{1} \rightarrow S^{1}$ be defined by

$$
E: \quad x \in S^{1} \rightarrow E(x)=k g(x) \bmod 1
$$

Let

$$
E_{\min }:=\min _{x}\left(\frac{d E}{d x}\right)(x)=k \min _{x}\left(\frac{d g}{d x}(x)\right)
$$

We will suppose that the function $g$ is such that

$$
E_{\min }>1
$$

so that $E$ is a uniform expanding map on $S^{1}$. The map $E$ is then a $k: 1$ map (i.e. every point $y$ has $k$ previous images $\left.x \in E^{-1}(y)\right)$. Let $\tau: S^{1} \rightarrow \mathbb{R}$ be a $C^{\infty}$ function, and define a map $f$ on $\mathbb{T}^{2}=S^{1} \times S^{1}$ by:

$$
f: \quad\left(\begin{array}{c}
x \\
s
\end{array}\right) \longmapsto\left(\begin{array}{lr}
x^{\prime}=E(x)=k g(x) & \bmod 1 \\
s^{\prime}=s+\frac{1}{2 \pi} \tau(x) & \bmod 1
\end{array}\right)
$$


The map $f$ is also a $k: 1$ map. The map $f$ is a very simple example of a compact group extension of the expanding map $E$ (see Dol02, Pes04, p.17]). It is also a special example of a partially hyperbolic map?. See figure 1.
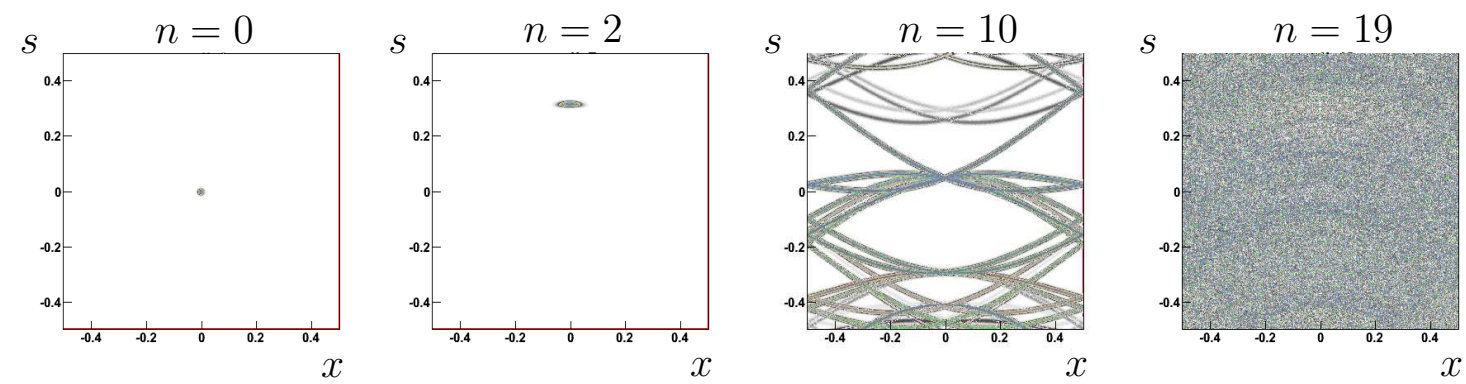

Figure 1: Numerical evolution of an initial small cloud of points on the torus $(x, s) \in \mathbb{T}^{2}$ under the map $f$, Eq.(3), at different time $n=0,2,10,19$. We have chosen here $E(x)=2 x$ and $\tau(x)=\cos (2 \pi x)$. The initial cloud of points is centered around the point $(0,0)$. For small time $n$, the cloud of point is transported in the vertical direction $s$ and spreads in the expanding horizontal direction $x$. Due to instability in $x$ and periodicity, the cloud fills the torus $S^{1} \times S^{1}$ for large time $n$. On the last image $n=19$, one observes an invariant absolutely continuous probability measure (called SRB measure, equal to the Lebesgue measure in our example). It reveals the mixing property of the map $f$ in this example.

\subsection{Transfer operator}

Instead of studying individual trajectories which have chaotic behavior, one prefer to study the evolutions of densities induced by the map $f$. This is the role of the Perron-Frobenius transfer operator $\hat{F}^{*}$ on $C^{\infty}\left(\mathbb{T}^{2}\right)$ given by:

$$
\left(\hat{F}^{*} \psi\right)(\mathbf{y})=\sum_{\mathbf{x} \in f^{-1}(\mathbf{y})} \frac{1}{\left|D_{\mathbf{x}} f\right|} \psi(\mathbf{x}), \quad \psi \in C^{\infty}\left(\mathbb{T}^{2}\right) .
$$

Indeed if the the function $\psi$ has its support in a vicinity of $x$ then the support of $\hat{F}^{*} \psi$ is in a vicinity of $y=f(x)$. To explain the Jacobian in the prefactor, one checks $]^{3}$ that $\int_{\mathbb{T}^{2}}\left(\hat{F}^{*} \psi\right)(\mathbf{y}) d \mathbf{y}=$ $\int_{\mathbb{T}^{2}} \psi(\mathbf{x}) d \mathbf{x}$, i.e. the total measure is preserved.

The operator $\hat{F}^{*}$ extends to a bounded operator on $L^{2}\left(\mathbb{T}^{2}, d \mathbf{x}\right)$. Its $L^{2}$-adjoint written $\hat{F}$ is defined by $\left(\hat{F}^{*} \psi, \varphi\right)_{L^{2}}=(\psi, \hat{F} \varphi)_{L^{2}}$, with the scalar product $(\psi, \varphi)_{L^{2}}:=\int_{\mathbb{T}^{2}} \bar{\psi}(\mathbf{x}) \varphi(\mathbf{x}) d \mathbf{x}$. One

\footnotetext{
${ }^{2} \mathrm{~A}$ even more general setting would be a $C^{\infty}$ map $f: M \rightarrow M$ on a compact Riemannian manifold $M$, which is supposed to be partially expanding, i.e., for any $m \in M$, the tangent space $T_{m} M$ decomposes continuously as

$$
T_{m} M=E_{u}(m) \oplus E_{0}(m)
$$
}

where $E_{u}(m)$ is a (non invariant) expanding direction (with respect to a Riemannian metric $g$ ):

$$
\left|D_{m} f\left(v_{u}\right)\right|_{g}>\left|v_{u}\right|_{g}, \quad \forall v_{u} \in E_{u}(m)
$$

and $E_{0}(m)$ the neutral direction: there exist a non zero global section $v_{0} \in C^{\infty}(T M)$ such that $v_{0}(m) \in E_{0}(m)$ and $D f\left(v_{0}\right)=v_{0}$. In our example (3), $M=S^{1} \times S^{1}$, the neutral section is $v_{0}=(0,1)$, and the expanding direction $E_{u}(m)$ is spanned by the vector $(1,0)$.

${ }^{3}$ Since $\mathbf{y}=f(\boldsymbol{x})$, then $d \mathbf{y}=\left|D_{\mathbf{x}} f\right| d \mathbf{x}$, and

$$
\int_{\mathbb{T}^{2}}\left(\hat{F}^{*} \psi\right)(\mathbf{y}) d \mathbf{y}=\sum_{\mathbf{x} \in f^{-1}(\mathbf{y})} \int_{\mathbb{T}^{2}} \frac{1}{\left|D_{\mathbf{x}} f\right|} \psi(\mathbf{x})\left|D_{\mathbf{x}} f\right| d \mathbf{x}=\int_{\mathbb{T}^{2}} \psi(\mathbf{x}) d \mathbf{x}
$$


checks easily that $\hat{F}$ has a simpler expression than $\hat{F}^{*}$ : it is the pull back operator, also called the Koopman operator, or Ruelle transfer operator and given by:

$$
(\hat{F} \psi)(\boldsymbol{x})=\psi(f(\boldsymbol{x}))
$$

\subsection{The reduced transfer operator}

The particular form of the map (3) allows some simplifications. Observe that for a function of the form

$$
\psi(x, s)=\varphi(x) e^{i 2 \pi \nu s}
$$

with $\nu \in \mathbb{Z}$ (i.e. a Fourier mode in $s$ ), then

$$
(\hat{F} \psi)(x, s)=\varphi(E(x)) e^{i \nu \tau(x)} e^{i 2 \pi \nu s} .
$$

Therefore the operator $\hat{F}$ preserves the following decomposition in Fourier modes:

$$
L^{2}\left(\mathbb{T}^{2}\right)=\bigoplus_{\nu \in \mathbb{Z}} \mathcal{H}_{\nu}, \quad \mathcal{H}_{\nu}:=\left\{\varphi(x) e^{i 2 \pi \nu s}, \quad \varphi \in L^{2}\left(S^{1}\right)\right\}
$$

The space $\mathcal{H}_{\nu}$ and $L^{2}\left(S^{1}\right)$ are unitary equivalent. For $\nu \in \mathbb{Z}$ given, the operator $\hat{F}$ restricted to the space $\mathcal{H}_{\nu} \equiv L^{2}\left(S^{1}\right)$, written $\hat{F}_{\nu}$ is

$$
\left(\hat{F}_{\nu} \varphi\right)(x):=\varphi(E(x)) e^{i \nu \tau(x)} \quad \varphi \in L^{2}\left(S^{1}\right) \equiv \mathcal{H}_{\nu}
$$

and with respect to the orthogonal decomposition (6), we can write:

$$
\hat{F}=\bigoplus_{\nu \in \mathbb{Z}} \hat{F}_{\nu}
$$

We will study the spectrum of this family of operators $\hat{F}_{\nu}$, with parameter $\nu \in \mathbb{Z}$, and consider more generally a real parameter $\nu \in \mathbb{R}$. We will see that the parameter $\nu$ is a semiclassical parameter, and $\nu \rightarrow \infty$ is the semiclassical limit. (if $\nu \neq 0, \nu=1 / \hbar$ in usual notations Mar02).

\section{Remarks:}

- For $\nu=0, \hat{F}_{0}$ has an obvious eigenfunction $\varphi(x)=1$, with eigenvalue 1. Except in special cases (e.g. $\tau=0$ ), there is no other obvious eigenvalues for $\hat{F}_{\nu}$ in $L^{2}\left(S^{1}\right)$.

\subsection{Main results on the spectrum of the transfer operator $\hat{F}_{\nu}$}

We first observe that by duality, the operator $\hat{F}_{\nu}$ defined in (7) extends to the distribution space $\mathcal{D}^{\prime}\left(S^{1}\right)$ :

$$
\hat{F}_{\nu}(\alpha)(\varphi)=\alpha\left(\hat{F}_{\nu}^{*}(\varphi)\right), \quad \alpha \in \mathcal{D}^{\prime}\left(S^{1}\right), \varphi \in C^{\infty}\left(S^{1}\right),
$$

where the $L^{2}$-adjoint $\hat{F}_{\nu}^{*}$ is given by

$$
\left(\hat{F}_{\nu}^{*} \varphi\right)(y)=\sum_{x \in E^{-1}(y)} \frac{e^{-i \nu \tau(x)}}{E^{\prime}(x)} \varphi(x), \quad \varphi \in C^{\infty}\left(S^{1}\right) .
$$

\footnotetext{
${ }^{4}$ Notice that the operator $\hat{F}_{\nu}$ appears to be a transfer operator for the expanding map $E$ with an additional weight function $e^{i \nu \tau(x)}$.
} 
Before giving the main results, remind that for $m \in \mathbb{R}$, the Sobolev space $H^{m}\left(S^{1}\right) \subset \mathcal{D}^{\prime}\left(S^{1}\right)$ consists in distributions (or continuous functions if $m>1 / 2$ ) such that their Fourier series $\hat{\psi}(\xi)$ satisfy $\sum_{\xi \in \mathbb{Z}}\left|\langle\xi\rangle^{m} \hat{\psi}(\xi)\right|^{2}<\infty$, with $\langle\xi\rangle:=\left(1+\xi^{2}\right)^{1 / 2}$. It can equivalently be written (Tay96a p.271).

$$
H^{m}\left(S^{1}\right):=\langle\hat{\xi}\rangle^{-m}\left(L^{2}\left(S^{1}\right)\right)
$$

with the differential operator $\hat{\xi}:=-i \frac{d}{d x}$.

The following theorem is well known [Rue86]. We will however provide a new proof based on semiclassical analysis.

\section{Theorem 1. Discrete spectrum of resonances.}

Let $m<0$. The operator $\hat{F}_{\nu}$ leaves the Sobolev space $H^{m}\left(S^{1}\right)$ invariant, and

$$
\hat{F}_{\nu}: H^{m}\left(S^{1}\right) \rightarrow H^{m}\left(S^{1}\right)
$$

is a bounded operator and can be written

$$
\hat{F}_{\nu}=\hat{R}+\hat{K}
$$

where $\hat{K}$ is a compact operator, and $\hat{R}$ has a small norm:

$$
\|\hat{R}\| \leq r_{m}:=\frac{1}{E_{m i n}^{|m|}} \sqrt{\frac{k}{E_{\min }}} .
$$

(the interesting situation is $m \ll 0$, since the norm $\|\hat{R}\|$ shrinks to zero for $m \rightarrow-\infty$ ).

Therefore, $\hat{F}_{\nu}$ has an essential spectral radius less than $r_{m}$, which means that $\hat{F}_{\nu}$ has discrete (eventually empty) spectrum of generalized eigenvalues $\lambda_{i}$ outside the circle of radius $r_{m}$ (see Tay960, prop. 6.9 p.499]). The eigenvalues $\lambda_{i}$ are called $\boldsymbol{R u e l l e ~ r e s o n a n c e s . ~ T o g e t h e r ~ w i t h ~ t h e i r ~ a s s o c i - ~}$ ated eigenspace, they do not depend on $m$ and are intrinsic to the transfer operator $\hat{F}_{\nu}$.

The following theorem is analogous to Theorem 1.1 in Tsu08a. However the approach and the proof we propose are different and rely on semiclassical analysis.

\section{Theorem 2. Spectral gap in the semiclassical limit.}

if the map $f$ is partially captive (definition given page 15) (and $m$ small enough), then the spectral radius of the operator $\hat{F}_{\nu}: H^{m}\left(S^{1}\right) \rightarrow H^{m}\left(S^{1}\right)$ does not depend on $m$ and satisfies in the semi-classical limit $\nu \rightarrow \infty$ :

$$
r_{s}\left(\hat{F}_{\nu}\right) \leq \frac{1}{\sqrt{E_{\min }}}+o(1)
$$

which is strictly smaller than 1 from (3).

\section{Remarks:}

- This remark concerns the regularity of the eigenfunctions of $\hat{F}_{\nu}$. Let $\lambda_{i}$ be a generalized eigenvalue of $\hat{F}_{\nu}$. Let $\varphi_{i}$ denotes a generalized eigenfunction of $\hat{F}_{\nu}$ associated to $\lambda_{i}$ (i.e. $\hat{F}_{\nu} \varphi_{i}=\lambda_{i} \varphi_{i}$ if $\lambda_{i}$ is an eigenvalue). Then $\varphi_{i}$ belongs to $H^{m}$ for any $m$ such that $m<m_{0}$ where $m_{0}$ is given by $r_{m_{0}}=\left|\lambda_{i}\right|$. 

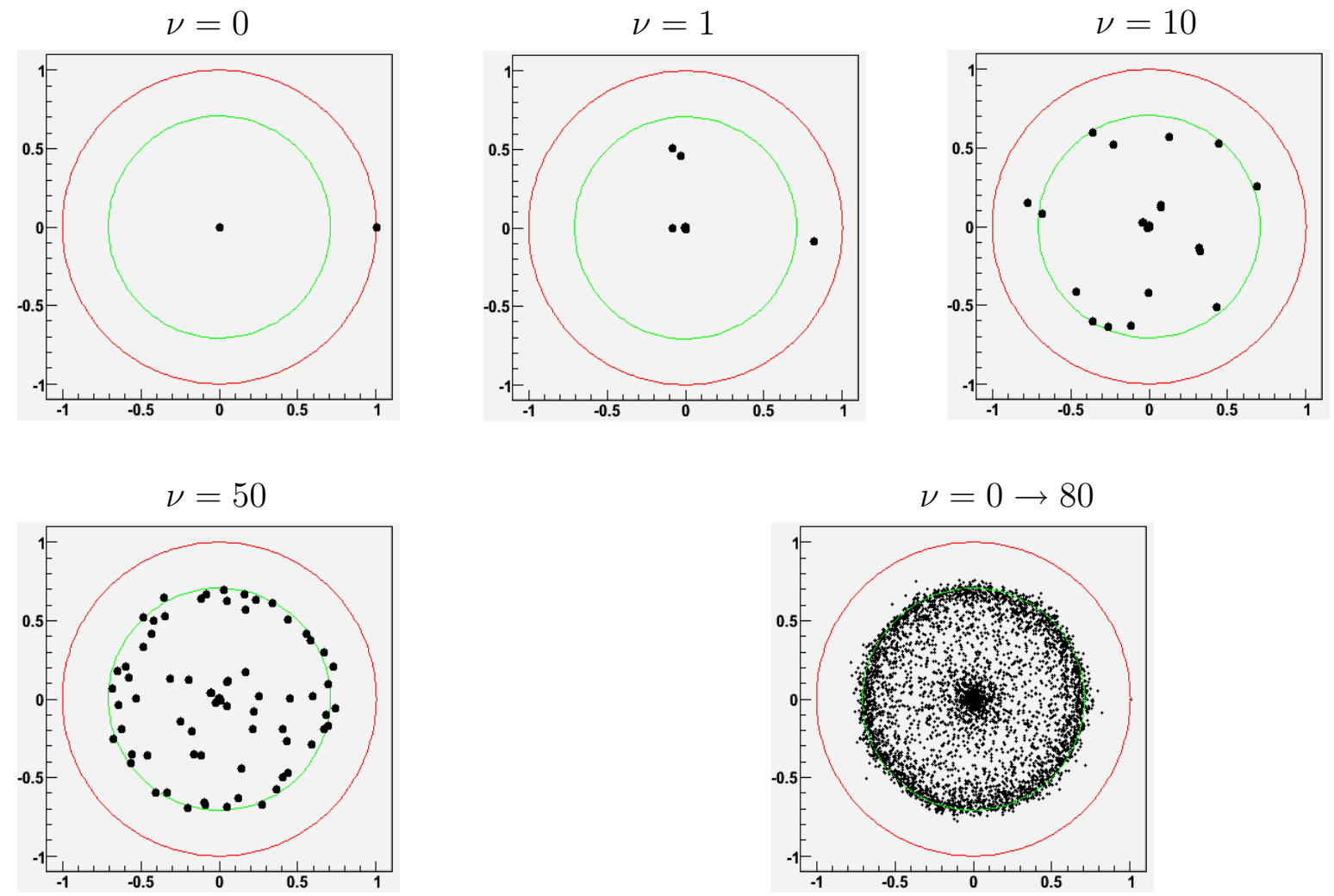

Figure 2: Black dots are numerical computation of the eigenvalues $\lambda_{i}$ of $\hat{F}_{\nu}$ for different values of $\nu \in \mathbb{N}$, and union of these in the last image. We have chosen here $E(x)=2 x$ i.e. $k=2$, and $\tau(x)=\cos (2 \pi x)$. The external red circle has radius 1 . The internal green circle has radius $1 / \sqrt{E_{\min }}=1 / \sqrt{2}$ and represents the upper bound given in Eq.(11). As $\nu \in \mathbb{R}$ moves continuously, the resonances move in a spectacular way. This can be seen on a movie on http://www-fourier. ujf-grenoble.fr/ faure/articles

- By duality we have similar spectral results for the Perron Frobenius operator $\hat{F}_{\nu}^{*}: H^{m}\left(S^{1}\right) \rightarrow$ $H^{m}\left(S^{1}\right)$ if $m>0$. The eigenvalues of $\hat{F}_{\nu}^{*}$ are $\bar{\lambda}_{i}$. We have seen that the generalized eigenfunctions of $\hat{F}_{\nu}$ belong to different Sobolev spaces $H^{m}\left(S^{1}\right)$ with $m<0$. Eq.(10) says that $m$ should satisfy $r_{m}<\left|\lambda_{i}\right|$, so $m \rightarrow-\infty$ as $\left|\lambda_{i}\right| \rightarrow 0$. The situation is simpler for the generalized eigenfunctions of $\hat{F}_{\nu}^{*}$ since they all belong to $\bigcap_{m>0} H^{m}=H^{\infty}=C^{\infty}\left(S^{1}\right)$.

- In the proof of Theorem 2 , we will obtain that a general bound for $r_{s}\left(\hat{F}_{\nu}\right)$ (with no hypothesis on $f$ ) is given by

$$
r_{s}\left(\hat{F}_{\nu}\right) \leq \frac{1}{\sqrt{E_{\min }}} \exp \left(\frac{1}{2} \lim _{n \infty}\left(\frac{\log \mathcal{N}(n)}{n}\right)\right)+o(1)
$$

where the function $\mathcal{N}(n)$ will be defined in Eq.(30). This bound is similar to the bound given in Tsu08a, Theorem 1.1] by M. Tsujii.

- In Tsu08a, Theorem 1.2] M. Tsujii shows that the partially captive property, i.e. $\lim _{n \infty}\left(\frac{\log \mathcal{N}(n)}{n}\right)=0$, is true for almost all functions $\tau$.

- From the definition of $\mathcal{N}(n)$ it is clear that $\mathcal{N}(n) \leq k^{n}$ hence $\exp \left(\frac{1}{2} \lim _{n \infty}\left(\frac{\log \mathcal{N}(n)}{n}\right)\right) \leq$ 
$\sqrt{k}$. Also from the definition of $E_{\text {min }}$, it is clear that $E_{\min } \leq k$ and therefore the upper bound in (12) is not sharp since it does not give the obvious bound $r_{s}\left(\hat{F}_{\nu}\right) \leq 1$ (see ERS08, corollary 2]). It is therefore tempting to conjecture that for almost all functions $\tau$ Eq.(11) can be replaced by:

$$
r_{s}\left(\hat{F}_{\nu}\right) \leq \frac{1}{\sqrt{k}}+o(1)
$$

- Notice that the above results say nothing about the existence of Ruelle resonances $\lambda_{i}$. The work of F. Naud Nau08 are the first results concerning the existence of resonances $\lambda_{i}$.

- One observes numerically that for large $\nu \in \mathbb{R}$, the eigenvalues $\lambda_{i}(\nu)$ repulse each other like eigenvalues of random complex matrices. (See a movie on http://www-fourier.ujf-grenoble. $\mathrm{fr} / \sim$ faure/articles). This suggests that many important questions of quantum chaos (e.g. the conjecture of Random Matrices Boh91) also concerns the Ruelle resonances of partially hyperbolic dynamics in the semiclassical limit.

- Remarks on numerical computation of the Ruelle resonances: one diagonalizes the matrix which expresses the operator $\hat{F}_{\nu}$ in Fourier basis $\varphi_{n}(x):=\exp (i 2 \pi n x), n \in \mathbb{Z}$. For the example of Figure 2 one gets $\left\langle\varphi_{n^{\prime}} \mid \hat{F} \varphi_{n}\right\rangle=e^{-i 2 \pi \frac{3}{4}\left(2 n-n^{\prime}\right)} J_{\left(2 n-n^{\prime}\right)}(\nu)$ where $J_{n}(x)$ is the Bessel function of first kind [AS54, 9.1.21 p 360]. Corollary 2 in [FR06] guaranties that the eigenvalues of the truncated matrix $|n|,\left|n^{\prime}\right| \leq N$ converges towards the Ruelle resonances as $N \rightarrow \infty$.

- One can proves AF09 that in the semi-classical limit $\nu \rightarrow \infty$, the number of Ruelle resonances $\lambda_{i}$ (counting multiplicities) outside a fixed radius $\lambda$ is bounded by a "Weyl law":

$$
\forall \lambda>0, \quad \sharp\left\{i \in \mathbb{N}, \quad \text { s.t. }\left|\lambda_{i}\right| \geq \lambda\right\} \leq\left(\frac{\nu}{2 \pi}\right) \mu(K)+o(\nu)
$$

where $\mu(K)$ is the Lebesgue measure of the trapped set $K$ defined later in Eq.(29). As usual in the semiclassical theory of non selfadjoint operators, see [jö90, SZ07, the Weyl law gives an upper bound for the density of resonances but no lower bound. See discussions in Non08, section 3.1$]$.

\subsection{Spectrum of $\hat{F}$ and dynamical correlation functions}

In this section, in order to give some "physical meaning" to the spectrum of $\hat{F}_{\nu}$, we recall relations between the spectral results of Theorems 1,2, and the evolution of correlation functions [Bal00]. This will allow us to interpret the evolution and convergence of clouds of points observed in Figure 1 .

Let $\nu \in \mathbb{Z}$. If $\psi_{1}, \psi_{2} \in C^{\infty}\left(S^{1}\right)$, the correlation function at time $n \in \mathbb{N}$ is defined by:

$$
C_{\psi_{2}, \psi_{1}}(n):=\left(\hat{F}_{\nu}^{* n} \psi_{2}, \psi_{1}\right)_{L^{2}}=\left(\psi_{2}, \hat{F}_{\nu}^{n} \psi_{1}\right)_{L^{2}}
$$

which represents the function $\psi_{2}$ evolved $n$ times by the Perron-Frobenius operator $\hat{F}_{\nu}^{*}$ and tested against the test function $\psi_{1}$.

The first spectral result of Theorem 1 implies that for any $\varepsilon>0$, and large $n$ (and assuming that the eigenvalues $\left(\lambda_{i, \nu}\right)_{i}$ of $\hat{F}_{\nu}$ are simple for short; see [FRS08 for a more extended discussion)

$$
C_{\psi_{2}, \psi_{1}}(n)=\sum_{\left|\lambda_{i, \nu}\right|>0} \lambda_{i, \nu}^{n} v_{i, \nu}\left(\bar{\psi}_{2}\right) w_{i, \nu}\left(\psi_{1}\right)+O_{\varepsilon}\left(\varepsilon^{n}\right)
$$

If the conclusion of Theorem 2 holds, this implies that for any $\rho$ such that $\frac{1}{\sqrt{E_{\text {min }}}}<\rho<1$, there exists $\nu_{0}$ such that for any $\nu \geq \nu_{0}$, all the eigenvalues of $\hat{F}_{\nu}$ are bounded: $\left|\lambda_{i, \nu}\right|<\rho<1, \forall i$. This gives an exponential decay of correlations for $n \rightarrow \infty$ in these space $\hat{F}_{\nu}$ :

$$
C_{\psi_{2}, \psi_{1}}(n)=\mathcal{O}\left(\rho^{n}\right)
$$


It is known that if the function $\tau$ is not a co-boundary (i.e. if the map $f$ is not equivalent to the trivial case $\tau=0$, as explained in Appendix A ) then the map $f$ is ergodic, which implies that all the eigenvalues $\lambda_{i, \nu}$ are strictly less than one: $\left|\lambda_{i, \nu}\right|<1, \forall \nu, \forall i$, except for $\lambda_{0,0}=1$ associated to the eigenfunction $\varphi(x)=1$. One deduces mixing property of the dynamics as observed in Figure 1.

\section{Proof of theorem $\mathbb{Q}$ on resonances spectrum}

In this proof, we follow closely the proof of theorem 4 in FRS08 although we deal here with expanding map instead of hyperbolic map, and this simplifies a lot, since we can work with ordinary Sobolev spaces and not anisotropic Sobolev spaces. Here $\nu \in \mathbb{Z}$ is fixed.

\subsection{Dynamics on the cotangent space $T^{*} S^{1}$}

The first step is to realize that in order to study the spectral properties of the transfer operator, we have to study the dynamics lifted on the cotangent space. This basic idea has already been exploited in FRS08.

In Eq.(11), the map $E: S^{1} \rightarrow S^{1}$ is a $k: 1$ map, which means that every point $y \in S^{1}$ has $k$ inverses denoted by $x_{\varepsilon} \in E^{-1}(y)$ and given explicitly by

$$
x_{\varepsilon}=E_{\varepsilon}^{-1}(y)=g^{-1}\left(\frac{y}{k}+\varepsilon \frac{1}{k}\right), \quad \text { with } \varepsilon=0, \ldots, k-1
$$

We will denote the derivative by $E^{\prime}(x):=d E / d x$.

Proposition 1. In Eq.(円) $\hat{F}_{\nu}$ is a Fourier integral operator (FIO) acting on $C^{\infty}\left(S^{1}\right)$.

The associated canonical transform on the cotangent space $(x, \xi) \in T^{*} S^{1} \equiv S^{1} \times \mathbb{R}$ is $k$-valued and given by:

$$
F(x, \xi)=\left\{F_{0}(x, \xi), \ldots, F_{k-1}(x, \xi)\right\}, \quad(x, \xi) \in S^{1} \times \mathbb{R}
$$

where for any $\varepsilon=0, \ldots, k-1$,

$$
F_{\varepsilon}: \begin{cases}x & \rightarrow x_{\varepsilon}^{\prime}=E_{\varepsilon}^{-1}(x)=g^{-1}\left(\frac{1}{k} x+\varepsilon \frac{1}{k}\right) \\ \xi & \rightarrow \xi_{\varepsilon}^{\prime}=E^{\prime}\left(x_{\varepsilon}^{\prime}\right) \xi=k g^{\prime}\left(x_{\varepsilon}^{\prime}\right) \xi\end{cases}
$$

Similarly the adjoint $\hat{F}^{*}$ is a FIO whose canonical transformation is $F^{-1}$. See figure 3 .

The proof is just that the operator $\varphi \rightarrow \varphi \circ E$ on $C^{\infty}\left(S^{1}\right)$ is one of the simplest example of Fourier integral operator, see [Mar02 example 2 p.150.

The term $e^{i \nu \tau(x)}$ in Eq.(7) does not contribute to the expression of $F$, since here $\nu$ is considered as a fixed parameter, and therefore $e^{i \nu \tau(x)}$ acts as a pseudodifferential operator (equivalently as a FIO whose canonical map is the identity).

The map $F$ is the map $E^{-1}$ lifted on the cotangent space $T^{*} S^{1}$ in the canonical way. Indeed, if we denote a point $(x, \xi) \in T^{*} S^{1} \equiv S^{1} \times \mathbb{R}$ then using the usual formula for differentials $y=$ $E(x)=k g(x) \Rightarrow d y=E^{\prime}(x) d x \Leftrightarrow \xi^{\prime}=E^{\prime}(x) \xi$, we deduce the above expression for $F$.

\section{Remarks}

- The physical meaning for $\hat{F}_{\varepsilon}$ being a Fourier Integral Operator is that if $\varphi_{(x, \xi)}$ is a wave packet "micro-localized" at position $(x, \xi) \in T^{*} S^{1}$ of phase space (this makes sense for $\xi \gg 1$, and means that the micro-support of $\varphi$ is $(x, \xi))$, then $\varphi^{\prime}:=\hat{F}_{\nu} \varphi_{(x, \xi)}$ will be a superposition 
of $k$ wave packets at positions $\left(x_{\varepsilon}^{\prime}, \xi_{\varepsilon}^{\prime}\right)=F_{\varepsilon}(x, \xi), \varepsilon=0, \ldots, k-1$, i.e. with a very restricted micro-support, controlled by the canonical map $F$.

- Observe that the dynamics of the map $F$ on $S^{1} \times \mathbb{R}$ has a quite simple property: the zero section $\left\{(x, \xi) \in S^{1} \times \mathbb{R}, \xi=0\right\}$ is globally invariant and any other point with $\xi \neq 0$ escapes towards infinity $(\xi \rightarrow \pm \infty)$ in a controlled manner:

$$
\left|\xi_{\varepsilon}^{\prime}\right| \geq E_{\min }|\xi|, \quad \forall \varepsilon=0, \ldots, k-1
$$

where $E_{\min }>1$ is given in (2).

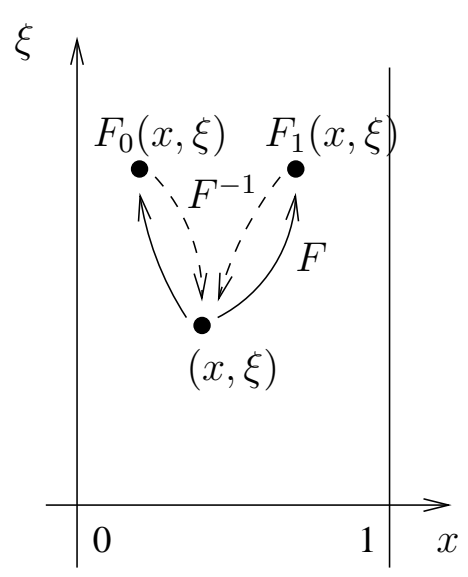

Figure 3: This figure is for $k=2$. The map $F=\left\{F_{0}, \ldots, F_{k-1}\right\}$ is $1: \mathrm{k}$, and its inverse $F^{-1}$ is $\mathrm{k}: 1$ on $T^{*} S^{1} \equiv S^{1} \times \mathbb{R}$.

\subsection{The escape function}

Let $m<0$ and define the $C^{\infty}$ function on $T^{*} S^{1}$ :

$$
A_{m}(x, \xi):=\langle\xi\rangle^{m} \quad \in S^{m}
$$

with $\langle\xi\rangle=\left(1+\xi^{2}\right)^{1 / 2} . A_{m}$ decreases with $|\xi|$ and belongs to the symbol class $S^{m}$.

Eq. (15) implies that the function $A_{m}$ decreases strictly along the trajectories of $F$ outside the zero section:

$\forall R>0, \forall|\xi|>R, \quad \forall \varepsilon=0, \ldots, k-1 \quad \frac{A_{m}\left(F_{\varepsilon}(x, \xi)\right)}{A_{m}(x, \xi)} \leq C^{|m|}<1, \quad$ with $C=\sqrt{\frac{R^{2}+1}{R^{2} E_{\min }+1}}<1$

Proof.

$$
\frac{A_{m}\left(F_{\varepsilon}(x, \xi)\right)}{A_{m}(x, \xi)}=\frac{\left(1+\xi^{2}\right)^{|m| / 2}}{\left(1+\left(\xi_{\varepsilon}^{\prime}\right)^{2}\right)^{|m| / 2}} \leq \frac{\left(1+\xi^{2}\right)^{|m| / 2}}{\left(1+E_{\min } \xi^{2}\right)^{|m| / 2}} \leq\left(\frac{1+R^{2}}{1+E_{\min } R^{2}}\right)^{|m| / 2}=C^{|m|}
$$

${ }^{5}$ See [Tay96b p.2. The class of symbols $S^{m}$, with order $m \in \mathbb{R}$, consists of functions on the cotangent space $A \in C^{\infty}\left(S^{1} \times \mathbb{R}\right)$ such that

$$
\left|\partial_{\xi}^{\alpha} \partial_{x}^{\beta} A\right|_{\infty} \leq C_{\alpha, \beta}\langle\xi\rangle^{m-|\alpha|}, \quad\langle\xi\rangle=\left(1+\xi^{2}\right)^{1 / 2}
$$


The symbol $A_{m}$ can be quantized into a pseudodifferential operator $\hat{A}_{m}$ (PDO for short) which is self-adjoint and invertible on $C^{\infty}\left(S^{1}\right)$ using the quantization rule (Tay96b p.2)

$$
(\hat{A} \varphi)(x)=\frac{1}{2 \pi} \int A(x, \xi) e^{i(x-y) \xi} \varphi(y) d y d \xi
$$

but in our simple case, this is very explicit: in Fourier space, $\hat{A}_{m}$ is simply the multiplication by $\langle\xi\rangle^{m}$.

Remind that the Sobolev space $H^{m}\left(S^{1}\right)$ is defined by (Tay96a p.271):

$$
H^{m}\left(S^{1}\right):=\hat{A}_{m}^{-1}\left(L^{2}\left(S^{1}\right)\right)
$$

The following commutative diagram

$$
\begin{aligned}
& L^{2}\left(S^{1}\right) \quad \stackrel{\hat{Q}_{m}}{\rightarrow} \quad L^{2}\left(S^{1}\right) \\
& \downarrow \hat{A}_{m}^{-1} \circlearrowleft \quad \downarrow \hat{A}_{m}^{-1} \\
& H^{m}\left(S^{1}\right) \quad \stackrel{\hat{F}_{\breve{ }}}{\rightarrow} H^{m}\left(S^{1}\right)
\end{aligned}
$$

shows that $\hat{F}_{\nu}: H^{m}\left(S^{1}\right) \rightarrow H^{m}\left(S^{1}\right)$ is unitary equivalent to

$$
\hat{Q}_{m}:=\hat{A}_{m} \hat{F}_{\nu} \hat{A}_{m}^{-1} \quad: L^{2}\left(S^{1}\right) \rightarrow L^{2}\left(S^{1}\right)
$$

We will therefore study the operator $\hat{Q}_{m}$. Notice that $\hat{Q}_{m}$ is defined a priori on a dense domain $\left(C^{\infty}\left(S^{1}\right)\right)$. Define

$$
\hat{P}:=\hat{Q}_{m}^{*} \hat{Q}_{m}=\hat{A}_{m}^{-1}\left(\hat{F}_{\nu}^{*} \hat{A}_{m}^{2} \hat{F}_{\nu}\right) \hat{A}_{m}^{-1}=\hat{A}_{m}^{-1} \hat{B} \hat{A}_{m}^{-1}
$$

where appears the operator

$$
\hat{B}:=\hat{F}_{\nu}^{*} \hat{A}_{m}^{2} \hat{F}_{\nu}
$$

The Egorov Theorem will help us to treat this operator (see [Tay96b p.24). This is a simple but crucial step in the proof: as explained in [FRS08], the Egorov theorem is the main Theorem used in order to establish both the existence of a discrete spectrum of resonances and properties of them. However there is a difference with FRS08: for the expanding map we consider here, the operator $\hat{F}_{\nu}$ is not invertible and the canonical map $F$ is $k$-valued. Therefore we have to state the Egorov theorem in an appropriate way (we restrict however the statement to our simple context).

Lemma 1. (Egorov theorem). $\hat{B}:=\hat{F}_{\nu}^{*} \hat{A}_{m}^{2} \hat{F}_{\nu}$ is a pseudo-differential operator with symbol in $S^{m}$ given by:

$$
B(x, \xi)=\left(\sum_{\varepsilon=0, \ldots, k-1} \frac{1}{E^{\prime}\left(x_{\varepsilon}^{\prime}\right)} A_{m}^{2}\left(F_{\varepsilon}(x, \xi)\right)\right)+R
$$

with $R \in S^{m-1}$ has a subleading order.

Proof. As we explained in Proposition 1, $\hat{F}_{\nu}$ and $\hat{F}_{\nu}^{*}$ are Fourier integral operators (FIO) whose canonical map are respectively $F$ and $F^{-1}$. The pseudodifferential operator (PDO) $\hat{A}_{m}$ can also be considered as a FIO whose canonical map is the identity. By composition we deduce that $\hat{B}=\hat{F}_{\nu}^{*} \hat{A}_{m}^{2} \hat{F}_{\nu}$ is a FIO whose canonical map is the identity since $F^{-1} \circ F=I d$. See figure 3 . Therefore $\hat{B}$ is a PDO. Using (8), (8) and (14) we obtain that the principal symbol of $\hat{B}$ is

$$
\sum_{\varepsilon=0, \ldots, k-1} \frac{1}{E^{\prime}\left(x_{\varepsilon}^{\prime}\right)} A_{m}^{2}\left(F_{\varepsilon}(x, \xi)\right)
$$


Remark: contrary to (19), $\hat{F}_{\nu} \hat{A}_{m} \hat{F}_{\nu}^{*}$ is not a PDO, but a FIO whose canonical map $F \circ F^{-1}$ is $k$-valued (see figure 3).

Now by theorem of composition of PDO (Tay96b p.11), (18) and (20) imply that $\hat{P}$ is a PDO of order 0 with principal symbol:

$$
P(x, \xi)=\frac{B(x, \xi)}{A_{m}^{2}(x, \xi)}=\left(\sum_{\varepsilon=0, \ldots, k-1} \frac{1}{E^{\prime}\left(x_{\varepsilon}^{\prime}\right)} \frac{A_{m}^{2}\left(F_{\varepsilon}(x, \xi)\right)}{A_{m}^{2}(x, \xi)}\right)
$$

The estimate (16) together with (2) give the following upper bound

$$
\forall|\xi|>R, \quad|P(x, \xi)| \leq C^{2|m|} \sum_{\varepsilon=0, \ldots, k-1} \frac{1}{E^{\prime}\left(x_{\varepsilon}^{\prime}\right)} \leq C^{2|m|} \frac{k}{E_{\min }}
$$

(This upper bound goes to zero as $m \rightarrow-\infty)$. From $L^{2}$-continuity theorem for PDO we deduce that for any $\alpha>0$ (see [FRS08] Lemma 38)

$$
\hat{P}=\hat{k}_{\alpha}+\hat{p}_{\alpha}
$$

with $\hat{k}_{\alpha}$ a smoothing operator (hence compact) and $\left\|\hat{p}_{\alpha}\right\| \leq C^{2|m|} \frac{k}{E_{m i n}}+\alpha$. If $\hat{Q}_{m}=\hat{U}|\hat{Q}|$ is the polar decomposition of $\hat{Q}_{m}$, with $\hat{U}$ unitary, then from $(18) \hat{P}=|\hat{Q}|^{2} \Leftrightarrow|\hat{Q}|=\sqrt{\hat{P}}$ and the spectral theorem (Tay96b p.75) gives that $|\hat{Q}|$ has a similar decomposition

$$
|\hat{Q}|=\hat{k}_{\alpha}^{\prime}+\hat{q}_{\alpha}
$$

with $\hat{k}_{\alpha}^{\prime}$ smoothing and $\left\|\hat{q}_{\alpha}\right\| \leq C^{|m|} \sqrt{\frac{k}{E_{m i n}}}+\alpha$, with any $\alpha>0$. Since $\|\hat{U}\|=1$ we deduce a similar decomposition for $\hat{Q}_{m}=\hat{U}|\hat{Q}|: L^{2}\left(S^{1}\right) \rightarrow L^{2}\left(S^{1}\right)$ and we deduce (9) and (10) for $\hat{F}_{\nu}: H^{m} \rightarrow H^{m}$. We also use the fact that $C \rightarrow 1 / E_{\min }$ for $R \rightarrow \infty$ in (16).

The fact that the eigenvalues $\lambda_{i}$ and their generalized eigenspaces do not depend on the choice of space $H^{m}$ is due to density of Sobolev spaces. We refer to the argument given in the proof of corollary 1 in [FRS08]. This finishes the proof of Theorem 1.

\section{Proof of theorem 2 on spectral gap}

We will follow steps by steps the same analysis as in the previous section. The main difference now is that in Theorem 2, $\nu \gg 1$ is a semi-classical parameter. In other words, we just perform a linear rescaling in cotangeant space: $\xi_{h}:=\hbar \xi$ with

$$
\hbar:=\frac{1}{\nu} \ll 1 \text {. }
$$

Therefore, our quantization rule for a symbol $A\left(x, \xi_{h}\right)$, Eq.17) writes now (see Mar02 p.22)

$$
(\hat{A} \varphi)(x)=\frac{1}{2 \pi \hbar} \int A(x, \xi) e^{i(x-y) \xi_{h} / \hbar} \varphi(y) d y d \xi_{h}
$$

For simplicity we will write $\xi$ for $\xi_{h}$ below.

\subsection{Dynamics on the cotangent space $T^{*} S^{1}$}

In Eq. (7) the multiplicative term $e^{i \nu \tau(x)}=e^{i \tau(x) / \hbar}$ acts now as a Fourier integral operator (FIO) and contributes to the transport (it was merely a P.D.O. for theorem 1 in Section 3 when $\nu$ was fixed). Its associated canonical transformation on $T^{*} S^{1}=S^{1} \times \mathbb{R}$ is $(x, \xi) \rightarrow\left(x, \xi+\frac{d \tau}{d x}(x)\right)$ (this is a direct consequence of stationary phase approximation in Fourier transform see Mar02, Examples 1,2 p.150]). We obtain: 
Proposition 2. In Eq. (1) $\hat{F}_{\nu}$ is a semi-classical Fourier integral operator acting on $C^{\infty}\left(S^{1}\right)$ (with semi-classical parameter $\hbar:=1 / \nu \ll 1$ ). The associated canonical transformation on the cotangent space $(x, \xi) \in T^{*} S^{1} \equiv S^{1} \times \mathbb{R}$ is $k$-valued and given by:

$$
\begin{aligned}
& F(x, \xi)=\left\{F_{0}(x, \xi), \ldots, F_{k-1}(x, \xi)\right\}, \quad(x, \xi) \in S^{1} \times \mathbb{R} \\
& F_{\varepsilon}: \begin{cases}x & \rightarrow x_{\varepsilon}^{\prime}=E^{-1}(x) \\
\xi & \rightarrow \xi_{\varepsilon}^{\prime}=E^{\prime}\left(x_{\varepsilon}^{\prime}\right) \xi+\frac{d \tau}{d x}\left(x_{\varepsilon}^{\prime}\right), \quad \varepsilon=0, \ldots, k-1\end{cases}
\end{aligned}
$$

Similarly $\hat{F}^{*}$ is a FIO whose canonical transformation is $F^{-1}$.

Notice that for simplicity we have kept the same notation for the canonical transformation $F$ although it differs from (14).

Since the map $F$ is $k$-valued, a trajectory is a tree. Let us precise the notation:

Definition 1. For $\varepsilon=\left(\ldots \varepsilon_{3}, \varepsilon_{2}, \varepsilon_{1}\right) \in\{0, \ldots, k-1\}^{\mathbb{N}^{*}}$, a point $(x, \xi) \in S^{1} \times \mathbb{R}$ and time $n \in \mathbb{N}^{*}$ let us denote:

$$
F_{\varepsilon}^{n}(x, \xi):=F_{\varepsilon_{n}} F_{\varepsilon_{n-1}} \ldots F_{\varepsilon_{1}}(x, \xi)
$$

For a given sequence $\varepsilon \in\{0, \ldots, k-1\}^{\mathbb{N}^{*}}$, a trajectory issued from the point $(x, \xi)$ is $\left\{F_{\varepsilon}^{n}(x, \xi), n \in \mathbb{N}\right\}$.

Notice that at time $n \in \mathbb{N}$, there are $k^{n}$ points issued from a given point $(x, \xi)$ :

$$
F^{n}(x, \xi):=\left\{F_{\varepsilon}^{n}(x, \xi), \quad \varepsilon \in\{0, \ldots, k-1\}^{n}\right\}
$$

The new term $\frac{d \tau}{d x}\left(x_{\varepsilon}^{\prime}\right)$ in the expression of $\xi_{\varepsilon}^{\prime}$, Eq. 24 , complicates significantly the dynamics near the zero section $\xi=0$. However a trajectory from an initial point with $|\xi|$ large enough still escape towards infinity:

Lemma 2. For any $1<\kappa<E_{\text {min }}$, there exists $R \geq 0$ such that for any $|\xi|>R$, any $\varepsilon=0, \ldots k-1$,

$$
\left|\xi_{\varepsilon}^{\prime}\right|>\kappa|\xi|
$$

Proof. From (24), one has $\xi_{\varepsilon}^{\prime}=E^{\prime}\left(x_{\varepsilon}^{\prime}\right) \xi+\tau^{\prime}\left(x_{\varepsilon}^{\prime}\right)$, so $\xi_{\varepsilon}^{\prime}-\kappa \xi=\left(E^{\prime}\left(x_{\varepsilon}^{\prime}\right)-\kappa\right) \xi+\tau^{\prime}\left(x_{\varepsilon}^{\prime}\right) \geq\left(E_{\min }-\kappa\right) \xi+$ $\min \tau^{\prime}>0$ if $\xi>-\frac{\min \tau^{\prime}}{\left(E_{m i n}-\kappa\right)} \geq 0$, and similarly $\xi_{\varepsilon}^{\prime}-\kappa \xi \leq\left(E_{\min }-\kappa\right) \xi+\max \tau^{\prime}<0$ if $\xi<-\frac{\max \tau^{\prime}}{\left(E_{m i n}-\kappa\right)}$.

We will denote the set:

$$
\mathcal{Z}:=S^{1} \times[-R, R]
$$

outside of which trajectories escape in a controlled manner (27). See figure 4.

\subsection{The trapped set $K$}

We will be interested now in the trajectories of $F$ which do not escape towards infinity. 
Definition 2. We define_the trapped set

$$
K:=\bigcap_{n \in \mathbb{N}}\left(F^{-1}\right)^{n}(\mathcal{Z})
$$

which contains points for which a trajectory at least does not escape towards infinity. See figure 怆. The definition of $K$ does not depend on the compact set $\mathcal{Z}$ (if $\mathcal{Z}$ is chosen large enough).

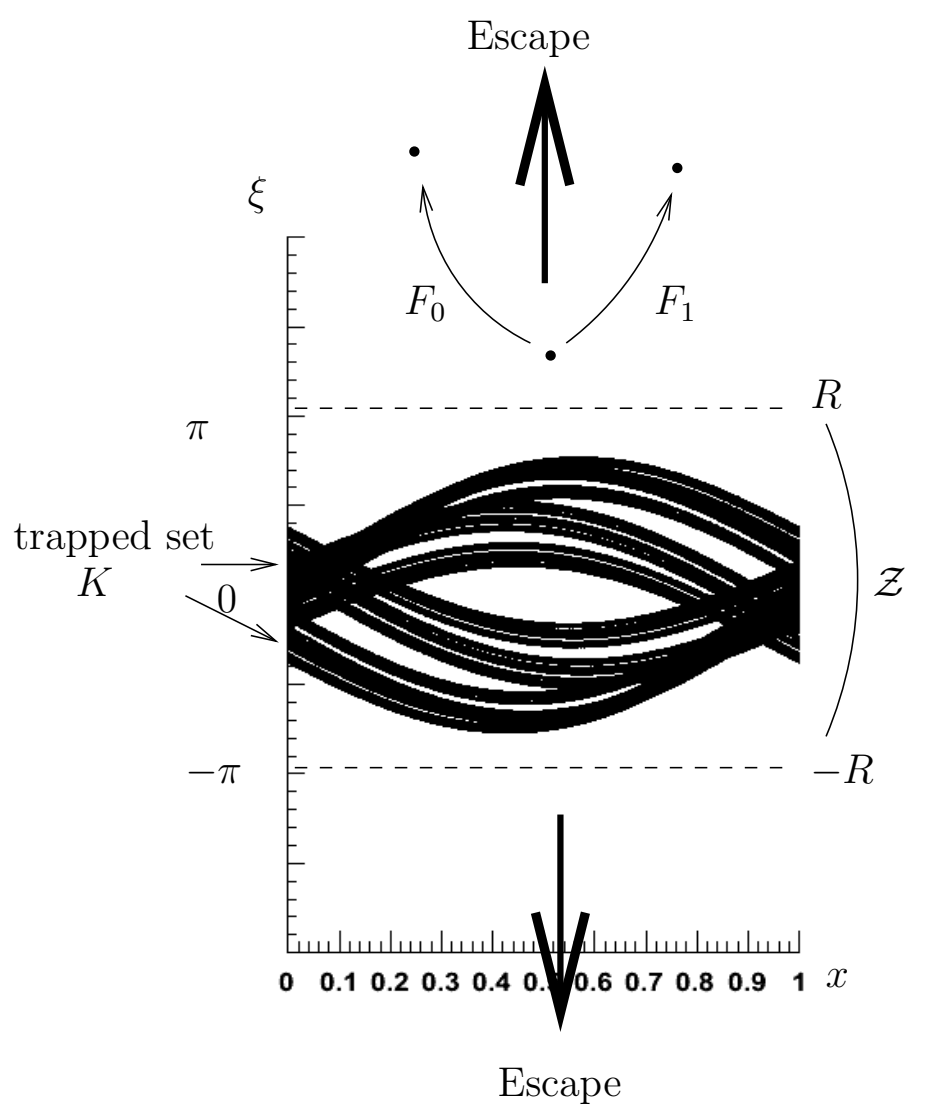

Figure 4: The trapped set $K$ in the cotangent space $S^{1} \times \mathbb{R}$. We have chosen here $E(x)=2 x$ and $\tau(x)=\cos (2 \pi x)$.

Since the map $F$ is multivalued, some trajectories may escape from the trapped set. We will need a characterization of how many such trajectories succeed to escape:

For $n \in \mathbb{N}$, let

$$
\mathcal{N}(n):=\max _{(x, \xi)} \sharp\left\{F_{\varepsilon}^{n}(x, \xi) \in \mathcal{Z}, \quad \varepsilon \in\{0, \ldots, k-1\}^{n}\right\}
$$

See Figure 5 for an illustration of $\mathcal{N}(n)$. Of course $\mathcal{N}(n) \leq k^{n}$. 
Definition 3. The map $F($ or $f)$ is partially captive if

$$
\frac{\log \mathcal{N}(n)}{n} \underset{n \rightarrow \infty}{\longrightarrow} 0
$$

This property is the hypothesis of Theorem .

\section{Remarks}

- "F partially captive" means that most of the trajectories escape from the trapped set $K$. See figure 5 . Another description of the trapped set $K$ and of the partially captive property will be given in Appendix B. Notice that the function $\mathcal{N}(n)$, Eq. (30) depends on the set $\mathcal{Z}$ but property (31) does not.

- If the function $\tau$ is trivial in (24), i.e. $\tau=0$, then obviously all the trajectories issued from a point $(x, \xi)$ on the line $\xi=0$ remains on this line (the trapped set). Therefore

$$
\sharp\left\{F_{\varepsilon}^{n}(x, \xi) \in \mathcal{Z}, \varepsilon \in\{0,1, \ldots, k-1\}^{n}\right\}=k^{n}
$$

and the map $F$ is not partially captive (but could be called "totally captive"). This is also true if the function $\tau$ is a "co-boundary", i.e. if $\tau(x)=\eta(E(x))-\eta(x)$ with $\eta \in C^{\infty}\left(S^{1}\right)$ as discussed in Appendix A.

- M. Tsujii has studied a dynamical system very similar to (24) in Tsu01, but this model is not volume preserving. He establishes there that the SRB measure on the trapped set is absolutely continuous for almost every $\tau$.

\subsection{The escape function}

Let $m<0$ and consider the $C^{\infty}$ function on $T^{*} S^{1}$ :

$$
\begin{aligned}
A_{m}(x, \xi) & :=\langle\xi\rangle^{m} \quad \text { for }|\xi|>R+\eta \\
& :=1 \quad \text { for } \xi \leq R
\end{aligned}
$$

where $\eta>0$ is small and with $\langle\xi\rangle:=\left(1+\xi^{2}\right)^{1 / 2}$. $A_{m}$ decreases with $|\xi|$ and belongs to the symbol class $S^{m}$.

Eq. (27) implies that the function $A_{m}$ decreases strictly along the trajectories of $F$ outside the trapped set (similarly to Eq.(16)):

$$
\forall|\xi|>R, \quad \forall \varepsilon=0, \ldots, k-1 \quad \frac{A_{m}\left(F_{\varepsilon}(x, \xi)\right)}{A_{m}(x, \xi)} \leq C^{|m|}<1, \quad \text { with } C=\sqrt{\frac{R^{2}+1}{\kappa R^{2}+1}}<1
$$

And for any point we have the general bound:

$$
\forall(x, \xi) \in T^{*} S^{1}, \quad \frac{A_{m}\left(F_{\varepsilon}(x, \xi)\right)}{A_{m}(x, \xi)} \leq 1 .
$$

Using the quantization rule (22), the symbol $A_{m}$ can be quantized giving a pseudodifferential operator $\hat{A}_{m}$ which is self-adjoint and invertible on $C^{\infty}\left(S^{1}\right)$. In our case $\hat{A}_{m}$ is simply a multiplication operator by $A_{m}(\xi)$ in Fourier space.

Let us consider the (usual) Sobolev space

$$
H^{m}\left(S^{1}\right):=\hat{A}_{m}^{-1}\left(L^{2}\left(S^{1}\right)\right)
$$




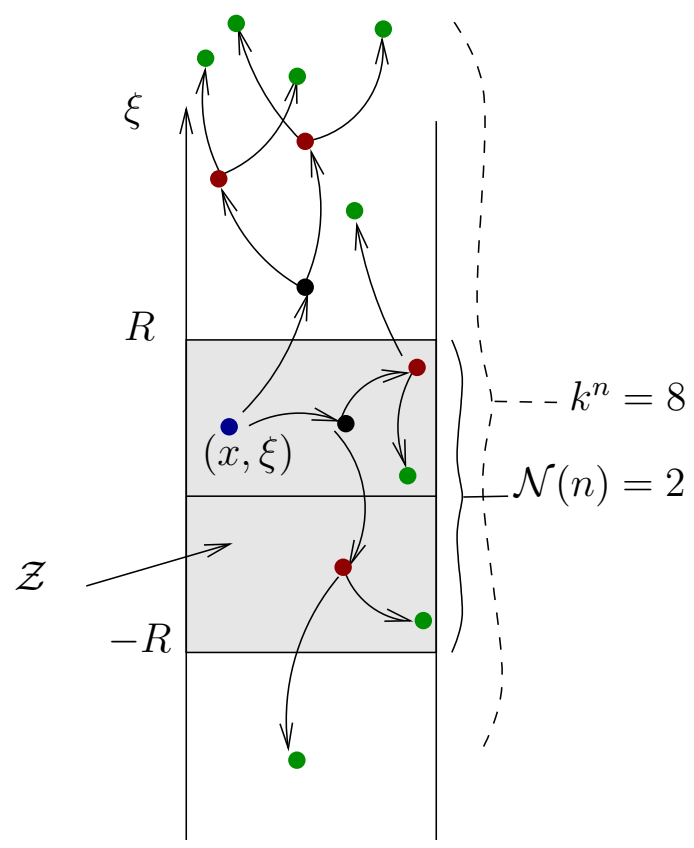

Figure 5: This Figure illustrates the trajectories $F_{\varepsilon}^{n}(x, \xi)$ issued from an initial point $(x, \xi)$. Here $k=2$ and $n=3$. The property for the map $F$ of being "partially captive" according to definition 3 is related to the number of points $\mathcal{N}(n)$ which do not escape from the compact zone $\mathcal{Z}$ after time $n$.

Then $\hat{F}_{\nu}: H^{m}\left(S^{1}\right) \rightarrow H^{m}\left(S^{1}\right)$ is unitary equivalent to

$$
\hat{Q}:=\hat{A}_{m} \hat{F}_{\nu} \hat{A}_{m}^{-1} \quad: L^{2}\left(S^{1}\right) \rightarrow L^{2}\left(S^{1}\right)
$$

Let $n \in \mathbb{N}^{*}$ (a fixed time which will be made large at the end of the proof) and define

$$
\hat{P}^{(n)}:=\hat{Q}^{* n} \hat{Q}^{n}=\hat{A}_{m}^{-1} \hat{F}_{\nu}^{* n} \hat{A}_{m}^{2} \hat{F}_{\nu}^{n} \hat{A}_{m}^{-1}
$$

Using Egorov theorem (the semi-classical version of Lemma (i) and Theorem of composition of PDO, we obtain that $\hat{P}^{(n)}$ is a PDO of order 0 with principal symbol

$$
P^{(n)}(x, \xi)=\left(\sum_{\varepsilon \in\{0, \ldots, k-1\}^{n}} \frac{1}{E_{n}^{\prime}(x)} \frac{A_{m}^{2}\left(F_{\varepsilon}^{n}(x, \xi)\right)}{A_{m}^{2}(x, \xi)}\right)
$$

where $E_{n}^{\prime}(x):=\prod_{j=1}^{n} E^{\prime}\left(E_{\varepsilon_{j}}^{-j}(x)\right)$ is the expanding rate of the trajectory at time $n$. Eq.(2) implies that $E_{n}^{\prime}(x) \geq E_{\min }^{n}$. Now we will bound this (positive) symbol from above, considering different cases for the trajectory $F_{\varepsilon}^{n}(x, \xi)$, as illustrated on Figure 5 .

1. If $(x, \xi) \notin \mathcal{Z}$ then (32) gives

$$
\frac{A^{2}\left(F_{\varepsilon}^{n}(x, \xi)\right)}{A^{2}(x, \xi)}=\frac{A^{2}\left(F_{\varepsilon}^{n}(x, \xi)\right)}{A^{2}\left(F_{\varepsilon}^{n-1}(x, \xi)\right)} \ldots \frac{A^{2}\left(F_{\varepsilon}(x, \xi)\right)}{A^{2}(x, \xi)} \leq\left(C^{2|m|}\right)^{n}
$$

therefore

$$
P^{(n)}(x, \xi) \leq \frac{k^{n}}{E_{\min }^{n}}\left(C^{2|m|}\right)^{n}
$$


2. If $(x, \xi) \in \mathcal{Z}$ but $F_{\varepsilon}^{n-1}(x, \xi) \notin \mathcal{Z}$ then $\frac{\left(A^{2} \circ F_{\varepsilon}^{n}\right)(x, \xi)}{\left(A^{2} \circ F_{\varepsilon}^{n-1}\right)(x, \xi)} \leq C^{2|m|}$ from (32). Using also (33) we have

$$
\frac{A^{2}\left(F_{\varepsilon}^{n}(x, \xi)\right)}{A^{2}(x, \xi)}=\frac{A^{2}\left(F_{\varepsilon}^{n}(x, \xi)\right)}{A^{2}\left(F_{\varepsilon}^{n-1}(x, \xi)\right)} \ldots \frac{A^{2}\left(F_{\varepsilon}(x, \xi)\right)}{A^{2}(x, \xi)} \leq C^{2|m|}
$$

3. In the other cases $\left((x, \xi) \in \mathcal{Z}\right.$ and $\left.F_{\varepsilon}^{n-1}(x, \xi) \in \mathcal{Z}\right)$ we can only use (33) to bound:

$$
\frac{A^{2}\left(F_{\varepsilon}^{n}(x, \xi)\right)}{A^{2}(x, \xi)} \leq 1
$$

From definition (30) we have

$$
\sharp\left\{F_{\varepsilon}^{n-1}(x, \xi) \in \mathcal{Z}, \quad \varepsilon \in\{0,1\}^{n}\right\} \leq \mathcal{N}(n-1) .
$$

For $(x, \xi) \in \mathcal{Z}$, we split the sum Eq.(35) accordingly to cases 1,2 or 3 above. Notice that $\left(C^{2|m|}\right)^{n} \leq$ $C^{2|m|}$. This gives

$$
P^{(n)}(x, \xi) \leq \frac{1}{E_{\min }^{n}}\left(\left(k^{n}-\mathcal{N}(n-1)\right) C^{2|m|}+\mathcal{N}(n-1)\right) \leq \mathcal{B}
$$

with the bound

Then

$$
\mathcal{B}:=\left(\frac{k}{E_{\min }}\right)^{n} C^{2|m|}+\frac{\mathcal{N}(n-1)}{E_{\text {min }}^{n}}
$$

$$
\limsup _{(x, \xi)}\left|P^{(n)}(x, \xi)\right| \leq \mathcal{B}
$$

With $L^{2}$ continuity theorem for pseudodifferential operators this implies that in the limit $\hbar \rightarrow 0$

$$
\left\|\hat{P}^{(n)}\right\| \leq \mathcal{B}+\mathcal{O}_{n}(\hbar)
$$

Polar decomposition of $\hat{Q}^{n}$ gives

$$
\left\|\hat{Q}^{n}\right\| \leq\left\|\hat{Q}^{n}\right\|=\sqrt{\left\|\hat{P}^{(n)}\right\|} \leq\left(\mathcal{B}+\mathcal{O}_{n}(\hbar)\right)^{1 / 2}
$$

Then for any $n$ the spectral radius of $\hat{Q}$ satisfies [RS72, p.192]

$$
r_{s}(\hat{Q}) \leq\left\|\hat{Q}^{n}\right\|^{1 / n} \leq\left(\mathcal{B}+\mathcal{O}_{n}(\hbar)\right)^{1 / 2 n}
$$

Also notice that

$$
\left(\frac{\mathcal{N}(n-1)}{E_{\text {min }}^{n}}\right)^{1 / 2 n}=\frac{1}{\sqrt{E_{\text {min }}}} \exp \left(\frac{1}{2 n} \log \mathcal{N}(n-1)\right)
$$

We let $\hbar \rightarrow 0$ first, and after $m \rightarrow-\infty$ giving $C^{|m|} \rightarrow 0$, and also we let $n \rightarrow \infty$. Then for $\hbar=1 / \nu \rightarrow 0$ we have 6 :

$$
r_{s}(\hat{Q}) \leq \sqrt{\frac{1}{E_{\min }} \exp \left(\lim _{n \infty} \inf \left(\frac{\log \mathcal{N}(n)}{n}\right)\right)}+o(1) .
$$

If we make the assumption that $F$ be partially captive, Eq.(31), we get that for $\hbar=1 / \nu \rightarrow 0$,

$$
r_{s}(\hat{Q}) \leq \frac{1}{\sqrt{E_{\text {min }}}}+o(1) \text {. }
$$

We have finished the proof of Theorem 2 .

\footnotetext{
${ }^{6}$ It can be shown that $\log \mathcal{N}(n)$ is sub-additive and therefore $\lim _{n \infty} \inf \left(\frac{\log \mathcal{N}(n)}{n}\right)=\lim _{n \infty}\left(\frac{\log \mathcal{N}(n)}{n}\right)$, RS72. p.217, ex.11]
} 


\section{A Equivalence classes of dynamics}

Let us make a simple and well known observation about equivalent classes of dynamics. The map $f$ we consider in Eq.(3) depends on $k \in \mathbb{N}$ and on the functions $E: S^{1} \rightarrow S^{1}, \tau: S^{1} \rightarrow \mathbb{R}$. To emphasize this dependence, we denote $f_{(E, \tau)}$. The transfer operator $(7)$ is also denoted by $\hat{F}_{(E, \tau)}$.

In this Appendix we characterize an equivalence class of functions $(E, \tau)$ such that in a given equivalence class the maps $f_{(E, \tau)}$ are $C^{\infty}$ conjugated together, the transfer operators $\hat{F}_{(E, \tau)}$ are also conjugated and the resonances spectrum are therefore the same.

Let $\eta: S^{1} \rightarrow \mathbb{R}$ be a smooth function. Let us consider the map $T: S^{1} \times S^{1} \rightarrow S^{1} \times S^{1}$ defined by

$$
T(x, s)=\left(x, s+\frac{1}{2 \pi} \eta(x)\right)
$$

Then using (3) one gets that:

$$
\left(T^{-1} \circ f_{(E, \tau)} \circ T\right)(x, s)=\left(E(x), s+\frac{1}{2 \pi}(\tau(x)+\eta(x)-\eta(E(x)))\right)
$$

Therefore

$$
\left(T^{-1} \circ f_{(E, \tau)} \circ T\right)=f_{(E, \zeta)}
$$

i.e. $f_{(E, \zeta)} \sim f_{(E, \tau)}$, with

$$
\zeta=\tau+(\eta-\eta \circ E) \text {. }
$$

The function $\tau$ has been modified by a "co-boundary term" ( KH95, p.100).

With (7) we also obtain that the transfer operator $\hat{F}_{(E, \zeta)}$ of $f_{(E, \zeta)}$ on $C^{\infty}\left(S^{1}\right)$ is given by

$$
\hat{F}_{(E, \zeta)}=\hat{\chi} \hat{F}_{(E, \tau)} \hat{\chi}^{-1}
$$

with the operator $\hat{\chi}: C^{\infty}\left(S^{1}\right) \rightarrow C^{\infty}\left(S^{1}\right)$ defined by:

$$
(\hat{\chi} \varphi)(x)=e^{i \nu \eta(x)} \varphi(x)
$$

Proof. $\left(\hat{\chi} \hat{F}_{(E, \tau)} \hat{\chi}^{-1} \varphi\right)(x)=\left(\varphi(E(x)) e^{-i \nu \eta(E(x))}\right) e^{i \nu \tau(x)} e^{i \nu \eta(x)}=\left(\hat{F}_{(E, \zeta)} \varphi\right)(x)$.

The conjugation (42) immediately implies that $\hat{F}_{(E, \zeta)}$ and $\hat{F}_{(E, \tau)}$ have the same spectrum of Ruelle resonances.

Observe that $\hat{\chi}$ is a O.I.F whose associated canonical transformation on $T^{*} S^{1} \equiv S^{1} \times \mathbb{R}$ is given by $(\nu \gg 1$ is considered as a semi-classical parameter):

$$
\chi:(x, \xi) \in\left(S^{1} \times \mathbb{R}\right) \rightarrow\left(x, \xi+\frac{d \eta}{d x}\right) \in\left(S^{1} \times \mathbb{R}\right) .
$$

Therefore at the level of canonical transforms on $T^{*} S^{1}$ :

$$
F_{(E, \zeta)}=\chi \circ F_{(E, \tau)} \circ \chi^{-1}
$$

The conjugation (43) implies in particular that the corresponding trapped sets (29) are related by

$$
K_{(E, \zeta)}=\chi\left(K_{(E, \tau)}\right)
$$

\section{B Description of the trapped set}

In this section we provide further description of the trapped set $K$ defined in Eq. 29) as well as the dynamics of the canonical map $F$ restricted on it. 


\section{B.1 Dynamics on the cover $\mathbb{R}^{2}$}

The dynamics of $F$ on the cylinder $T^{*} S^{1}=S^{1} \times \mathbb{R}$ has been defined in Eq.(24). This is a multivalued map. It is convenient to consider the lifted dynamics on the cover $\mathbb{R}^{2}$ which is a diffeomorphism given by

$$
\tilde{F}: \begin{cases}x & \rightarrow x^{\prime}=E^{-1}(x) \\ \xi & \rightarrow \xi^{\prime}=E^{\prime}\left(x^{\prime}\right) \xi+\frac{d \tau}{d x}\left(x^{\prime}\right)\end{cases}
$$

where $E=k g: \mathbb{R} \rightarrow \mathbb{R}$ is the map (17) lifted on $\mathbb{R}$. It is invertible from (2). Let us suppose for simplicity that $E(0)=0$.

One easily establish the following properties of the map $\tilde{F}$, illustrated on figure 6:

- The point

$$
I:=\left(0, \xi_{I}\right):=\left(0,-\frac{\tau^{\prime}(0)}{\left(E^{\prime}(0)-1\right)}\right)
$$

is the unique fixed point of $\tilde{F}$. It is hyperbolic with unstable manifold

$$
W_{u}=\{(0, \xi), \xi \in \mathbb{R}\}
$$

and stable manifold

$$
W_{s}=\{(x, S(x)), x \in \mathbb{R}\}
$$

where the $C^{\infty}$ function $S(x)$ is defined by the following co-homological equation, deduced directly from (44)

$$
S\left(E^{-1}(x)\right)=E^{\prime}\left(E^{-1}(x)\right) S(x)+\tau^{\prime}\left(E^{-1}(x)\right), \quad S(0)=\xi_{I} .
$$

The first equation can be written as

$$
S(x)=\frac{1}{E^{\prime}\left(E^{-1}(x)\right)}\left(S\left(E^{-1}(x)\right)-\tau^{\prime}\left(E^{-1}(x)\right)\right)
$$

and recursively we deduce that

$$
S(x)=-\sum_{p=1}^{\infty} \frac{1}{E^{\prime(-p)}(x)} \tau^{\prime}\left(E^{(-p)} x\right)
$$

where

$$
x_{(-p)}:=E^{(-p)}(x):=(\underbrace{E^{-1} \circ \ldots \circ E^{-1}}_{p})(x)
$$

and

$$
E^{\prime(-p)}(x):=E^{\prime}\left(x_{-p}\right) \ldots E^{\prime}\left(x_{-2}\right) E^{\prime}\left(x_{-1}\right)
$$

is the product of derivatives. In the case of $E(x)=2 x$, one gets simply

$$
S(x)=-\sum_{p=1}^{\infty} \frac{1}{2^{p}} \tau^{\prime}\left(\frac{x}{2^{p}}\right)
$$

- If $\mathcal{P}:(x, \xi) \in \mathbb{R}^{2} \rightarrow(x \bmod 1, \xi) \in S^{1} \times \mathbb{R} \equiv T^{*} S^{1}$ denotes the projection, then trapped set $K$, defined in Eq.(29) is obtained by wrapping the stable manifold around the cylinder and taking the closure:

$$
K=\overline{\mathcal{P}\left(W_{s}\right)}
$$

Compare Figures 6 and Figure 6 . 
- If $X_{0}=\left(x_{0}, \xi_{0}\right) \in \mathbb{R}^{2}$ is an initial point on the plane, and $\mathcal{P}\left(X_{0}\right)$ denotes its image on the cylinder, then at time $n \in \mathbb{N}$, the $k^{n}$ evolutions of the point $\mathcal{P}\left(X_{0}\right)$ under the map $F^{n}$ are the images of the evolutions $\tilde{F}^{n}\left(X_{k}\right)$ of the translated points $X_{p}=X_{0}+(p, 0)$, with $p=0 \rightarrow k^{n}-1$ :

$$
F^{n}\left(\mathcal{P}\left(X_{0}\right)\right)=\left\{\mathcal{P}\left(\tilde{F}^{n}\left(X_{p}\right)\right), X_{p}=X_{0}+(p, 0), \quad p \in\left[0, k^{n}[\}\right.\right.
$$

and more precisely, using notation of Eq.(25) for these points, one has the relation:

$$
F_{\varepsilon}^{n}\left(\mathcal{P}\left(X_{0}\right)\right)=\mathcal{P}\left(\tilde{F}^{n}\left(X_{p}\right)\right)
$$

where $\varepsilon$ is the number $p$ written in base $k$ :

$$
\varepsilon=\varepsilon_{n-1} \ldots \varepsilon_{1} \varepsilon_{0}=p_{\text {base } k} \in\{0, \ldots, k-1\}^{n}
$$

Figure 7 illustrates this correspondence.

- For an initial point $X_{0}=\left(x_{0}, \xi_{0}\right) \in \mathbb{R}^{2}$, then $X_{n}=\left(x_{n}, \xi_{n}\right)=\tilde{F}^{n}\left(X_{0}\right)$ satisfies

$$
x_{n}=E^{(-n)}\left(x_{0}\right), \quad \xi_{n}-S\left(x_{n}\right)=\left(E^{\prime(-n)}\left(x_{0}\right)\right)\left(\xi_{0}-S\left(x_{0}\right)\right)
$$

with $E^{(-n)}(x), E^{\prime(-n)}(x)$ given by (47), (48). Hence

$$
\left|\xi_{n}-S\left(x_{n}\right)\right| \geq E_{\min }^{n}\left|\xi_{0}-S\left(x_{0}\right)\right|
$$

This last inequality describes how fast the trajectories above or below the separatrix $W_{s}$ escape towards infinity on Figure 6 .

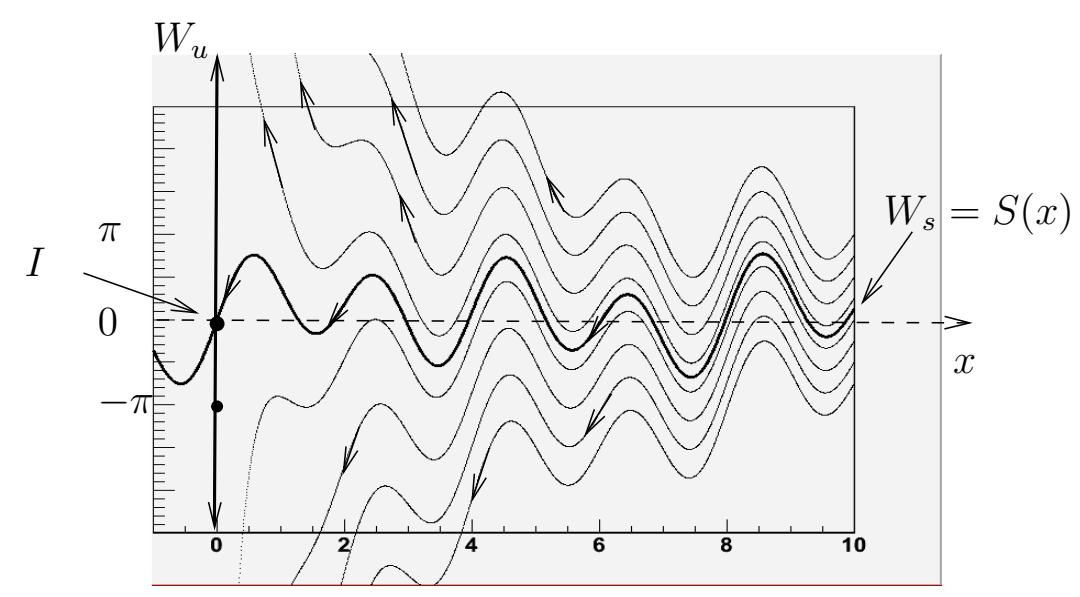

Figure 6: The fixed point $I=\left(0, \xi_{0}\right)$, the stable manifold $W_{s}$ and unstable manifold $W_{u}$ of the lifted map $\tilde{F}$, Eq.(44), in the example $E(x)=2 x, \tau(x)=\cos (2 \pi x)$.

\section{B.2 Partially captive property}

Here we rephrase the property of partial captivity, Definition 3, in terms of a property on the separatrix function $S(x)$ defined in Eq.(45) and given in Eq. (46).

For simplicity, we consider from now on the simple model with a linear expanding map $E(x)=$ $k x$. 

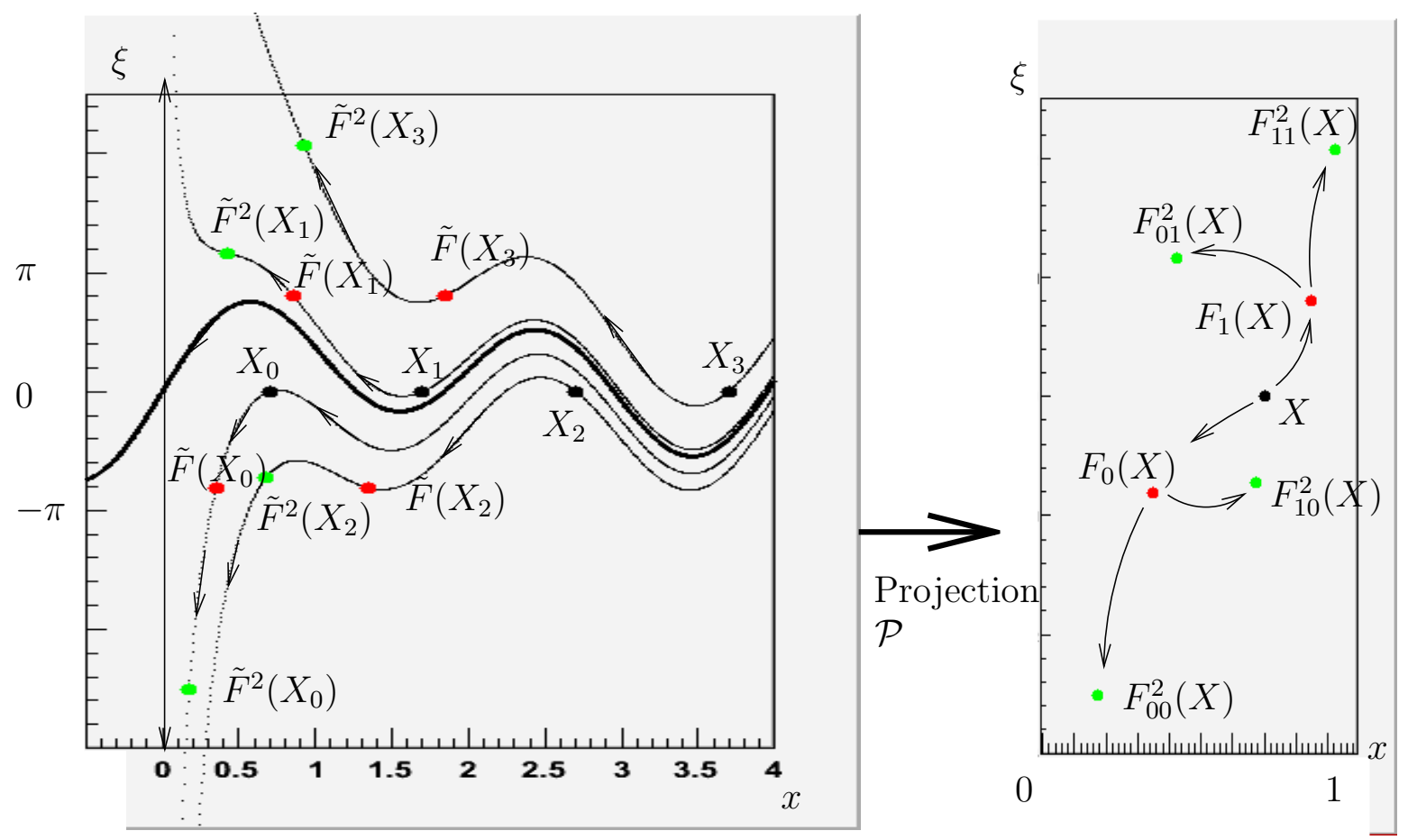

Figure 7: This picture shows how the dynamics of a point $X=\mathcal{P}\left(X_{0}\right) \in S^{1} \times \mathbb{R}$ under the map $F$ is related by Eq. (51) to the dynamics of its lifted images $X_{k}=X_{0}+(k, 0)$ under $\tilde{F}$ on the cover $\mathbb{R}^{2}$.

Proposition 3. For $n \in \mathbb{N}$, and $\tilde{R}>0$, let

$$
\tilde{\mathcal{N}}_{\tilde{R}}(n)=\max _{(x, \xi) \in \mathbb{R}^{2}} \sharp\left\{p \in \left[0, k^{n}\left[, \quad|\xi-S(x+p)| \leq \frac{\tilde{R}}{k^{n}}\right\}\right.\right.
$$

Then the map $F$ is partially captive (see definition page 15) if and only if

$$
\lim _{n \rightarrow \infty} \frac{\log \tilde{\mathcal{N}}_{\tilde{R}}(n)}{n}=0
$$

for $\tilde{R}$ large enough.

Proof. From (30) and using (51), (52) one gets

$$
\begin{aligned}
\mathcal{N}(n) & =\max _{(x, \xi)} \sharp\left\{F_{\varepsilon}^{n}((x, \xi)) \in \mathcal{Z}, \quad \varepsilon \in\{0, \ldots, k-1\}^{n}\right\} \\
& =\max _{(x, \xi)} \sharp\left\{\left|\xi_{n, p}\right| \leq R, \quad p \in\left[0, k^{n}[\}\right.\right.
\end{aligned}
$$

with $\left(x_{n, p}, \xi_{n, p}\right):=\tilde{F}^{n}((x+p, \xi))$ given by $x_{n, p}=\frac{x+p}{k^{n}}$ and $\xi_{n, p}=S\left(x_{n, p}\right)+k^{n}(\xi-S(x+p))$. Therefore

$$
\left|\xi_{n, p}\right| \leq R \Leftrightarrow\left|\frac{S\left(x_{n, p}\right)}{k^{n}}+\xi-S(x+p)\right| \leq \frac{R}{k^{n}}
$$


But $S$ is a bound function on $\mathbb{R},|S(x)| \leq S_{\max }$. Therefore $\left|\frac{S\left(x_{n, p}\right)}{k^{n}}+\xi-S(x+p)\right| \leq \frac{R}{k^{n}} \Longrightarrow$ $|\xi-S(x+p)| \leq \frac{\tilde{R}}{k^{n}}$ with some $\tilde{R}>0$, and conversely. This implies that (54) is equivalent to (31).

\section{B.3 Fractal aspect of the Trapped set}

The characterization Eq. (53) concerns the discrete set of points $S(x+m), m \in \mathbb{Z}$. From Eq.(50) these points are the slice of the trapped set $K=\cup_{x \in S^{1}} K_{x}$ :

$$
K_{x}=\overline{\{S(x+m), m \in \mathbb{Z}\}}
$$

For simplicity, we consider from now on the simple model with $E(x)=2 x$, and $\tau(x)=$ $\cos (2 \pi x)$.

From Eq.(49), these points are given by

$$
S(x+m)=\sum_{p=1}^{\infty} \frac{2 \pi}{2^{p}} \sin \left(\frac{2 \pi}{2^{p}}(x+m)\right)=\Im\left(\sum_{p=1}^{\infty} \frac{2 \pi}{2^{p}} \exp \left(\frac{i 2 \pi}{2^{p}}(x+m)\right)\right)
$$

Therefore the slice $K_{x}$ is the projection on the imaginary axis of the following set:

$$
\begin{gathered}
K_{x}^{c}=\overline{\left\{S^{c}(x+m), m \in \mathbb{Z}\right\}} \\
S^{c}(x+m)=\sum_{p=1}^{\infty} \frac{2 \pi}{2^{p}} e^{i 2 \pi \frac{1}{2^{p}(x+m)}}
\end{gathered}
$$

On Figure 8 we observe that $K_{x}^{c}$ is a fractal set. Compare Figure 8 with Figure 1.

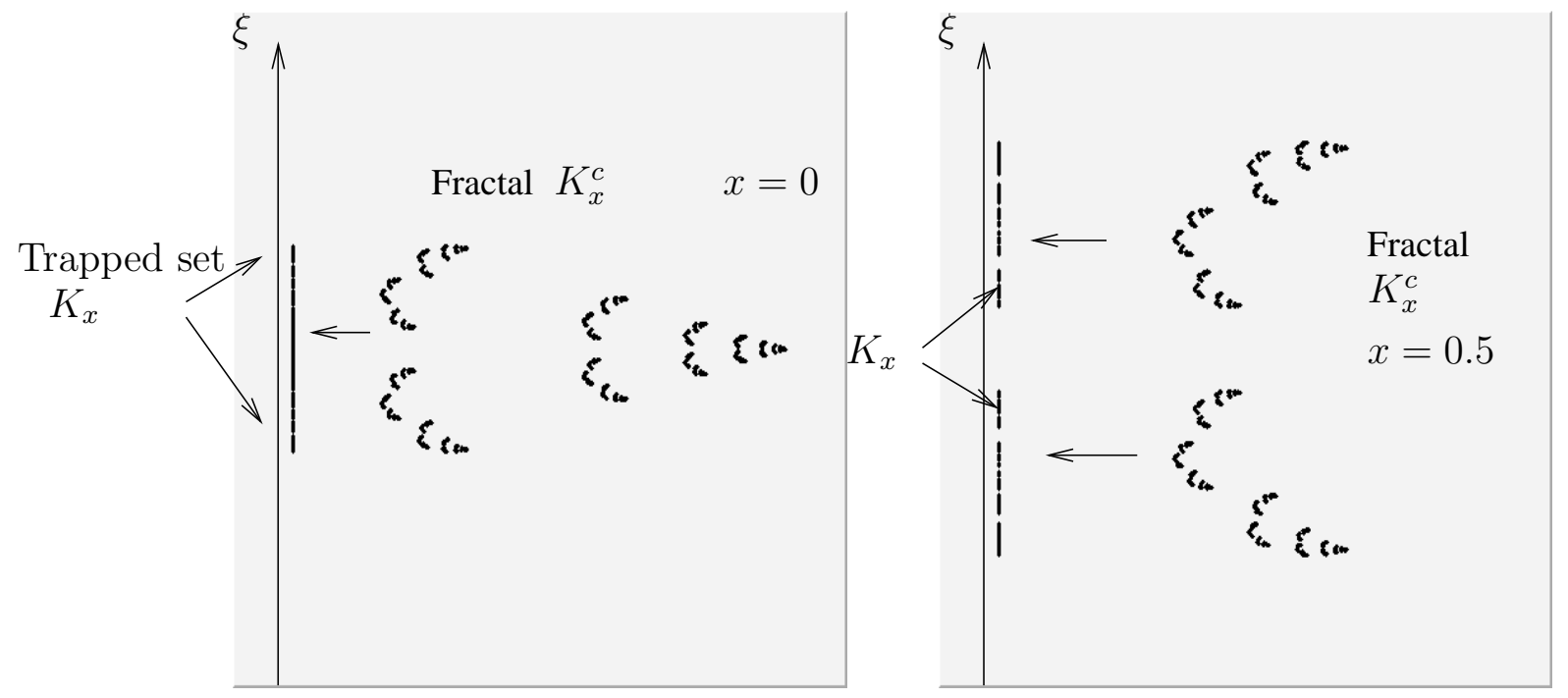

Figure 8: This picture represents the set $K_{x}^{c} \subset \mathbb{C}$ defined by Eq.(55). The trapped set $K$ at position $x$ is obtained by the projection on the imaginary axis $K_{x}=\Im\left(K_{x}^{c}\right)$. On the web page http://www-fourier.ujf-grenoble.fr/ faure/articles one can observe the motion of the fractal $K_{x}^{c}$ as $x \in \mathbb{R}$ increases smoothly.

\section{References}

[AF09] J.F. Arnoldi and F. Faure. work in progress. 2009. 
[AS54] M. Abramowitz and I. A. Stegun. Handbook of mathematical functions. Dover, 1954.

[Bal00] V. Baladi. Positive transfer operators and decay of correlations. Singapore: World Scientific, 2000.

[Bal05] V. Baladi. Anisotropic Sobolev spaces and dynamical transfer operators: $C^{\infty}$ foliations. Kolyada, S. (ed.) et al., Algebraic and topological dynamics. Proceedings of the conference, Bonn, Germany, May 1-July 31, 2004. Providence, RI: American Mathematical Society (AMS). Contemporary Mathematics, pages 123-135, 2005.

[BKL02] M. Blank, G. Keller, and C. Liverani. Ruelle-Perron-Frobenius spectrum for Anosov maps. Nonlinearity, 15:1905-1973, 2002.

[Boh91] O. Bohigas. Random matrix theories and chaotic dynamics. Chaos and Quantum Physics, Proceedings of the Les Houches Summer School (1989), 45:87-199, 1991.

[BT07] V. Baladi and M. Tsujii. Anisotropic Hölder and Sobolev spaces for hyperbolic diffeomorphisms. Ann. Inst. Fourier, 57:127-154, 2007.

[Dol98] D. Dolgopyat. On decay of correlations in Anosov flows. Ann. of Math. (2), 147(2):357$390,1998$.

[Dol02] D. Dolgopyat. On mixing properties of compact group extensions of hyperbolic systems. Israel J. Math., 130:157-205, 2002.

[FMT07] M. Field, I. Melbourne, and A. Török. Stability of mixing and rapid mixing for hyperbolic flows. Ann. of Math. (2), 166(1):269-291, 2007.

[FR06] F. Faure and N. Roy. Ruelle-pollicott resonances for real analytic hyperbolic map. http://arxiv.org/abs/nlin.CD/0601010. Nonlinearity, 19:1233-1252, 2006.

[FRS08] F. Faure, N. Roy, and J. Sjöstrand. A semiclassical approach for anosov diffeomorphisms and ruelle resonances. Open Math. Journal. (arXiv:0802.1780), 1:35-81, 2008.

[GL05] S. Gouzel and C. Liverani. Banach spaces adapted to Anosov systems. Ergodic Theory and dynamical systems, 26:189-217, 2005.

[HS86] B. Helffer and J. Sjöstrand. Résonances en limite semi-classique. (resonances in semiclassical limit). Memoires de la S.M.F., 24/25, 1986.

[KH95] A. Katok and B. Hasselblatt. Introduction to the Modern Theory of Dynamical Systems. Cambridge University Press, 1995.

[Liv04] C. Liverani. On contact Anosov flows. Ann. of Math. (2), 159(3):1275-1312, 2004.

[Liv05] C. Liverani. Fredholm determinants, anosov maps and ruelle resonances. Discrete and Continuous Dynamical Systems, 13(5):1203-1215, 2005.

[Mar02] A. Martinez. An Introduction to Semiclassical and Microlocal Analysis. Universitext. New York, NY: Springer, 2002.

[Nau08] F. Naud. Entropy and decay of correlations for real analytic semi-flows. preprint, 2008.

[Non08] S. Nonnenmacher. Some open questions in 'wave chaos'. Nonlinearity, 21(8):T113-T121, 2008.

[Pes04] Y. Pesin. Lectures on Partial Hyperbolicity and Stable Ergodicity. European Mathematical Society, 2004.

[RS72] M. Reed and B. Simon. Mathematical methods in physics, vol I : Functional Analysis. Academic press, New York, 1972. 
[Rue78] D. Ruelle. Thermodynamic formalism. The mathematical structures of classical equilibrium. Statistical mechanics. With a foreword by Giovanni Gallavotti. Reading, Massachusetts: Addison-Wesley Publishing Company., 1978.

[Rue86] D. Ruelle. Locating resonances for axiom A dynamical systems. J. Stat. Phys., 44:281292, 1986 .

[Sjö90] J. Sjöstrand. Geometric bounds on the density of resonances for semiclassical problems. Duke Math. J., 60(1):1-57, 1990.

[SZ07] J. Sjöstrand and M. Zworski. Fractal upper bounds on the density of semiclassical resonances. Duke Math. J., 137:381-459, 2007.

[Tay96a] M. Taylor. Partial differential equations, Vol I. Springer, 1996.

[Tay96b] M. Taylor. Partial differential equations, Vol II. Springer, 1996.

[Tsu01] M. Tsujii. Fat solenoidal attractors. Nonlinearity, 14(5):1011-1027, 2001.

[Tsu08a] M. Tsujii. Decay of correlations in suspension semi-flows of angle-multiplying maps. Ergodic Theory and Dynamical Systems, 28:291-317, 2008.

[Tsu08b] M. Tsujii. Quasi-compactness of transfer operators for contact Anosov flows. arXiv:0806.0732v2 [math.DS], 2008. 